\title{
Exercício Concorrente: Análise do Efeito Agudo da Ordem de Execução Sobre o Gasto Energético Total
}

\section{Concurrent Exercise: Analysis of the Acute Effect of the Performance Order on the Total Energy Expenditure}

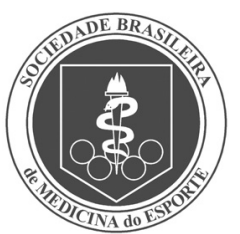

Artigo Original
Valéria Leme Gonçalves Panissa',2,

Rômulo Cássio de Moraes

Bertuzzi ${ }^{1,2}$,

Fábio Santos de Lira,

Ursula Ferreira Júlio ${ }^{1,2}$

Emerson Franchini ${ }^{2}$

1. Curso de Educação Física

- Centro de Ciências Biológicas e da

Saúde da Universidade Presbiteriana Mackenzie.

2. Escola de Educação Física e Esporte da Universidade de São

Paulo

3. Instituto de Ciências Biomédicas da Universidade de São Paulo.

Endereço para correspondência: Valéria Leme Gonçalves Panissa. Rua Dona Herminda Borba Attuy, 252

- Pq. São Domingos - 05123-010

- São Paulo, SP - Brasil.

E-mail: valeriapanissa@gmail.com

Submetido em 01/02/2008 Versão final recebida em 25/04/2008 Aceito em 24/10/2008

\begin{abstract}
RESUMO
O objetivo do presente estudo foi analisar o efeito da ordem de execução dos exercícios de força e aeróbio sobre o gasto energético total na sessão. Para isso, dez homens tiveram seu consumo de oxigênio medido continuamente durante as seguintes sessões: aeróbio-força (AF) e força-aeróbio (FA). O exercício aeróbio consistiu de 30 minutos de corrida na esteira a 90\% da velocidade do limiar anaeróbio. A sessão de força foi composta de quatro exercícios, na qual os participantes realizavam três séries de 12 repetições a $70 \%$ de 1 RM. A concentração de lactato sanguíneo [La] foi mensurada após cada exercício de força e nos minutos 10, 20 e 30 do exercício aeróbio. A [La] durante a execução do exercício aeróbio permaneceu maior $(p<0,05)$ na situação FA quando comparado com a AF aos 10 ( $\left.F A=5,1 \pm 1,3 \mathrm{mmol}^{-1}{ }^{-1} ; A F=3,2 \pm 1,0 \mathrm{mmol} . \mathrm{I}^{-1}\right), 20$ (FA=4,2 $\pm 1,0 \mathrm{mmol} . \mathrm{I}^{-1} ; \mathrm{AF}=3,0 \pm 0,9 \mathrm{mmol} . \mathrm{I}^{-1}$ ) e 30 minutos ( $\mathrm{FA}=3,9 \pm 1,3 \mathrm{mmol} . \mathrm{I}^{-1} ; \mathrm{AF}=3,4 \pm 1,1 \mathrm{mmol} . \mathrm{I}^{-1}$ ). O gasto energético total não diferiu entre as ordens de exercício ( $F A=2.793 \pm 811 \mathrm{~kJ} ; A F=2.893 \pm 903 \mathrm{~kJ} ; p>0,05)$, indicando que a ordem de execução não afetou significativamente o gasto energético.
\end{abstract}

Palavras-chave: aeróbio, treinamento de força, exercício concorrente, gasto energético.

\begin{abstract}
The aim of present study was to analyze the effect of aerobic and strength exercises order on the total energy expenditure in an exercise session. Ten male subjects had their $\mathrm{VO}_{2}$ continuously measured during two sessions: aerobic-resistance (AR) and resistance-aerobic (RA) to estimate caloric expenditure. The aerobic session was a 30-min treadmill run at 90\% of anaerobic threshold velocity. The strength exercise session had four exercises, where participants performed three sets of 12 repetitions at $70 \%$ of 1RM. Blood lactate concentration (LA) was measured after each strength exercise and at 10,20 and 30 min during the aerobic exercise. LA during aerobic exercise was higher $(\mathrm{p}<0.05)$ in RA order when compared to $A R$ at 10 (RA $=5.1 \pm 1.3 \mathrm{mmol}^{-\mathrm{I}^{-1} \text {; }}$

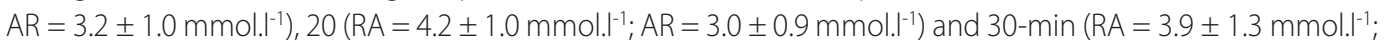
$\left.A R=3.4 \pm 1.1 \mathrm{mmol}^{-1}{ }^{-1}\right)$. Total caloric expenditure did not differ between exercise orders (RA $=2793 \pm 811 \mathrm{~kJ}$; $A R=2893 \pm 903 \mathrm{~kJ} ; p>0.05)$, indicating that performance order did not affect energy expenditure.
\end{abstract}

Keywords: aerobic, strength training, concurrent exercise, energy expenditure.

\section{INTRODUÇÃO}

A inatividade física tem sido apontada frequentemente como um dos principais fatores responsáveis pelo desencadeamento de doenças crônico-degenerativas ${ }^{(1)}$. Nesse sentido, a realização periódica de exercícios físicos parece atuar como um agente não-farmacológico no controle de uma parte dessas doenças ${ }^{(2,3)}$. Logo, os exercícios de força e aeróbio estão entre as principais tarefas empregadas nos estudos que analisaram a eficácia de programas de condicionamento físico que objetivam a manutenção da saúde ${ }^{(4)}$. Em linhas gerais, acredita-se que, ao se exercitar utilizando isoladamente os exercícios predominantemente aeróbios, há diminuiç̧ão dos estoques de gordura corpora|(5), ao passo que o treinamento de força induz o aumento da massa magra ${ }^{(6)}$.

Por sua vez, o American College of Sports Medicine tem proposto que, para manutenção da saúde e da forma física adequada, os indivíduos adultos saudáveis devem ter gasto energético (GE) proveniente da prática de atividade física de aproximadamente 300-500kcal por dia ou 700-2.000kcal por semana. Além disso, essa instituição também propôs que os exerć́- cios de força deveriam ser realizados em duas ou três sessões semanais, as quais seriam compostas por oito ou dez exercícios diferentes, com as intensidades que permitissem a execução entre oito e 12 repetições( ${ }^{(4)}$.

A realização dos exercícios de força e aeróbio na mesma sessão de condicionamento físico é uma prática comum e tem sido frequentemente denominada de treinamento concorrente $(\mathrm{TC})^{(7)}$. Em relação ao desempenho nos dois tipos de exercício, embora os achados sejam discordantes, acredita-se que a ordem de execução do treinamento interfira na tarefa principal(7,8). Por outro lado, estudos prévios analisaram o comportamento do consumo de oxigênio $\left(\dot{V}_{2}\right)$ durante a execução de uma atividade aeróbia realizada posteriormente a uma sessão de exercícios de força e detectaram que a realização dos exercícios aeróbios imediatamente após uma sessão de treinamento resistido pode resultar em valores maiores do GE quando comparada com sua execução isoladamente ${ }^{(9,10)}$. Contudo, $\operatorname{Scott}^{(11)}$ demonstrou que a mensuração do gasto energético durante uma sessão de treinamento de força pode ser subestimada quando não se levam em conta as participações dos metabolismos anaeróbios. 
Dessa forma, o objetivo do presente estudo foi analisar agudamente o efeito da manipulação da ordem de execução dos exercícios aeróbios e de força sobre o GE. Para tanto, admitiu-se que o $\dot{V}_{2}$, as concentrações sanguíneas de lactato ([La]]) e a fase rápida do excesso do consumo de oxigênio após o exercício (EPOC rápido $)$ representam as contribuições dos metabolismos energéticos aeróbio, anaeróbio lático e anaeróbio alático, respectivamente ${ }^{(11,12)}$.

\section{MÉTODOS}

\section{Amostra e desenho experimental}

Dez sujeitos do sexo masculino e fisicamente ativos concordaram em participar voluntariamente do presente estudo após leitura e assinatura do termo de consentimento informado. Eles foram submetidos a quatro sessões de coletas de dados, as quais foram realizadas em um intervalo máximo de três semanas e com um intervalo mínimo de 48 horas. Na primeira e na segunda sessões foram realizados os testes para determinação do limiar anaeróbio e da força máxima dinâmica em quatro aparelhos de musculação, respectivamente. Esses testes foram realizados para a determinação das intensidades dos treinamentos aeróbio e de força, respectivamente. Na terceira e na quarta sessões os treinamentos de força e aeróbio foram efetivamente realizados, sendo que a ordem de execução desses exercícios foi randomizada. Durante as sessões três e quatro, os participantes tiveram seu consumo de oxigênio mensurado continuamente, além da medida da concentração de lactato sanguíneo em repouso, durante a recuperação de cada exercício de força e em intervalos de 10 minutos durante o exercício aeróbio. Foi recomendado aos indivíduos que mantivessem sua dieta rotineira antes de todas as sessões e que não realizassem nenhum outro tipo de atividade física intensa nas 24h que antecederam os testes. $\mathrm{O}$ estudo foi previamente aprovado pelo comitê de ética local, respeitando as normas internacionais de experimentação com humanos.

\section{Determinação do consumo de oxigênio de pico e do limiar anaeróbio}

Para determinação do consumo de oxigênio de pico $\left(\dot{V}_{O_{2 p i c o}}\right)$ e do limiar anaeróbio (Lan), foi realizada uma adaptação do método descrito por Heck et al. ${ }^{(13)}$. Brevemente, os sujeitos foram submetidos a um teste escalonado em uma esteira rolante da marca Aegean (modelo 6200), com velocidade inicial de $5 \mathrm{~km} \cdot \mathrm{h}^{-1}$ e incrementos de $1 \mathrm{~km} \cdot \mathrm{h}^{-1} \mathrm{a}$ cada três minutos. Durante todo o teste houve a mensuração do $\dot{\mathrm{V}}_{2}$ e da frequência cardíaca (FC), ao passo que as [La-] foram medidas ao final de cada estágio. O Lan foi detectado mediante a velocidade correspondente à concentração fixa de $3,\left.5 \mathrm{mmo} \cdot\right|^{-1}$ das [La-]. A partir dessa intensidade, a velocidade foi aumentada em $0,5 \mathrm{~km} \cdot \mathrm{h}^{-1}$ a cada minuto até a exaustão voluntária, sendo que as amostras de sangue não eram mais coletadas. Essa adaptação foi realizada com o intuito de se atingir o tempo ideal para a mensuração do $\dot{\mathrm{V}} \mathrm{O}_{2 \text { pico }}{ }^{(14)}$. $\mathrm{O} \dot{\mathrm{V}} \mathrm{O}_{2 \text { pico }}$ foi estabelecido pela média aritmética do $\dot{\mathrm{V}}_{2}$ dos 30 segundos finais do último estágio em que o indivíduo conseguiu manter-se em exercício por um minuto. A FC de pico ( $\left.F C_{\text {pico }}\right)$ foi estabelecida pelo maior valor da FC obtido nesse estágio.

\section{Força dinâmica máxima}

A força dinâmica máxima foi determinada mediante a carga máxima em que o sujeito foi capaz de executar apenas um movimento (1RM) nos exercícios de supino inclinado, puxador costas, cadeira extensora e mesa flexora dos joelhos. Para tanto, foram seguidas as recomendações da Sociedade Americana de Fisiologia do Exercício(15). Além disso, não houve o controle do ritmo de execução das repetições no intuito de se aproximar ao máximo do modo como esses exercícios são executados em sessões típicas. Nesse sentido, os indivíduos iniciaram o teste realizando um aquecimento geral de cinco minutos. Subsequentemente, eles executaram uma série de oito repetições com a intensidade de 50\% da carga de 1RM estimada, seguida por outra série de três repetições a 70\% de 1RM também estimada. Os levantamentos para estabelecer 1RM foram execuções únicas com carga progressivamente mais pesada até a fadiga voluntária. O intervalo de descanso foi de três a cinco minutos e o número de tentativas não foi superior a cinco.

\section{Treinamento de força e aeróbio}

Os exercícios que constituíram o treinamento de força foram executados na seguinte ordem: supino inclinado, cadeira extensora, puxador costas e mesa flexora dos joelhos. Adotando as recomendações feitas por Melby et al. ${ }^{(16)}$, esses exercícios foram sistematizados em três séries, com a intensidade de $70 \%$ de 1RM e com 12 repetições ou até a exaustão voluntária. Os intervalos de tempo entre as séries foram de dois minutos. As amostras de sangue para a mensuração das [La] foram obtidas após a realização dos exercícios em cada aparelho.

Em relação ao exercício aeróbio, os sujeitos se exercitaram na esteira rolante na intensidade correspondente a 90\% do Lan durante 30 minutos. As coletas de sangue foram feitas nos minutos 10, 20 e 30. A mensuração das [La-] também foi realizada em repouso, independentemente do exercício que iniciou a sessão de treinamento físico.

\section{Aquisição dos dados metabólicos e cálculo do gasto energético}

A mensuração do $\dot{V}_{2}$ foi realizada por meio de um analisador de gases portátil de circuito aberto (VO2000, Imbrasport), o qual teve a sua calibração feita antes de cada teste de acordo com as recomendações do fabricante desse equipamento. A frequência de aquisição da medida do $\dot{V}_{2}$ foi constituída por uma média móvel de três expirações e posteriormente alisada para intervalos de 20 segundos. As microamostras de sangue obtidas do lóbulo da orelha foram imediatamente analisadas em um lactímetro portátil (Accusport - Roche) para a determinação das [La'].

A contribuição do metabolismo aeróbio foi calculado a partir do $\dot{\mathrm{V}}_{2}$ mensurado durante os exercícios, ao passo que a participação do metabolismo anaeróbio alático foi assumida como sendo o $\mathrm{EPOC}_{\text {rápido }}{ }^{(12,17)}$. Em nosso estudo adotamos a medida do $\dot{\mathrm{V}}_{2}$ nos períodos de recuperação para representar o $E P O C_{\text {rápido, }}$ haja vista que a maior parte da ressíntese dos fosfatos de alta energia ocorre entre os dois-três minutos de recuperação ${ }^{(11,18)}$. Para a estimativa da energia anaeróbia lática admitiu-se que o valor de $\left.1 \mathrm{mmol} \cdot\right|^{-1}$ dos valores delta das [La-] equivale a $3 \mathrm{ml}$ de $\mathrm{O}_{2} \cdot \mathrm{kg}^{-1}$ de massa corporal(19). Para os três sistemas energéticos foi admitido o equivalente calórico de $20,9 \mathrm{~kJ} \cdot \cdot^{-1}$ de $\mathrm{O}_{2}$. O gasto energético total foi estabelecido a partir do somatório dos três metabolismos energéticos.

\section{ANÁLISES ESTATÍSTICAS}

Os dados foram analisados estatisticamente mediante o programa SPSS (13.0) e expressos em médias \pm desvios padrão. Foi utilizada a análise de variância a dois fatores (situação e tempo) com medidas repetidas para verificar a influência da ordem dos exercícios no desempenho das tarefas e nas respostas fisiológicas. Quando constatada diferença, foi conduzido um teste de Tukey. A comparação do gasto calórico total nas duas situações foi feita através de um teste t de Student para amostras dependentes. Para todas as análises foi adotado o nível de significância de 5\% ( $<<0,05)$. 


\section{RESULTADOS}

A idade cronológica, as características físicas e funcionais dos participantes estão presentes na tabela 1.

Tabela 1. Valores da idade cronológica, das características físicas e funcionais dos sujeitos $(n=10)$.

\begin{tabular}{l|c}
\hline Idade (anos) & $24 \pm 5$ \\
\hline Peso $(\mathrm{kg})$ & $73,0 \pm 11,4$ \\
\hline Estatura $(\mathrm{cm})$ & $176,0 \pm 8,0$ \\
\hline$\dot{\text { VOO}}{ }_{2}$ pico $\left(\mathrm{m}^{\prime} \cdot \mathrm{kg}^{-1} \cdot \mathrm{min}^{-1}\right)$ & $55,0 \pm 5,3$ \\
\hline FCpico $(\mathrm{bpm})$ & $193 \pm 10$ \\
\hline 1 RM supino inclinado $(\mathrm{kg})$ & $63 \pm 13$ \\
\hline 1 RM cadeira extensora $(\mathrm{kg})$ & $52 \pm 10$ \\
\hline 1 RM puxador costas $(\mathrm{kg})$ & $68 \pm 13$ \\
\hline 1 RM mesa flexora $(\mathrm{kg})$ & $41 \pm 9$ \\
\hline
\end{tabular}

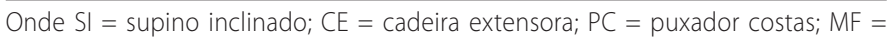
mesa flexora.
Na figura 1 está presente um exemplo típico da resposta do $\dot{\mathrm{V}}_{2}$ durante a realização do exercício aeróbio (painel A) e de força (painel B) mediante as diferentes ordens de execução. Não foram constatadas diferenças significativas do $\dot{\mathrm{V}}_{2}$ no exercício aeróbio mediante a ordem de execução dos exercícios ( $p>0,05$ ), ao passo que a média do $\mathrm{VO}_{2}$ permaneceu estatisticamente mais elevada durante a ordem AF $\left(11,5 \pm 3,6 \mathrm{ml} \cdot \mathrm{kg}^{-1} \cdot \mathrm{min}^{-1}\right)$ na execução do supino inclinado quando

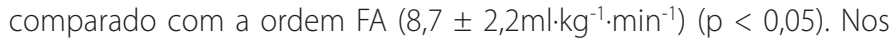
demais exercícios de força as diferenças constatadas não foram significativas $(p>0,05)$

Na figura 2 estão presentes os valores das [La] durante a realização dos exercícios aeróbios (painel A) e de força (painel B) em virtude das ordens de execução. Foi constatada diferença significativa $(p<0,05)$ nas [La] durante a realização do exercício aeróbio, no qual a [La־] permaneceu mais elevada nos minutos 10, 20 e 30 da ordem FA em comparação com a ordem inversa. Diferentemente do exercício aeróbio, não foram constatadas diferenças significativas das [La'] durante o treinamento de força $(p>0,05)$. Além disso, também não foram detectadas diferenças significativas da ordem de execução dos exercícios sobre o gasto energético total (figura 3) $(p>0,05)$.

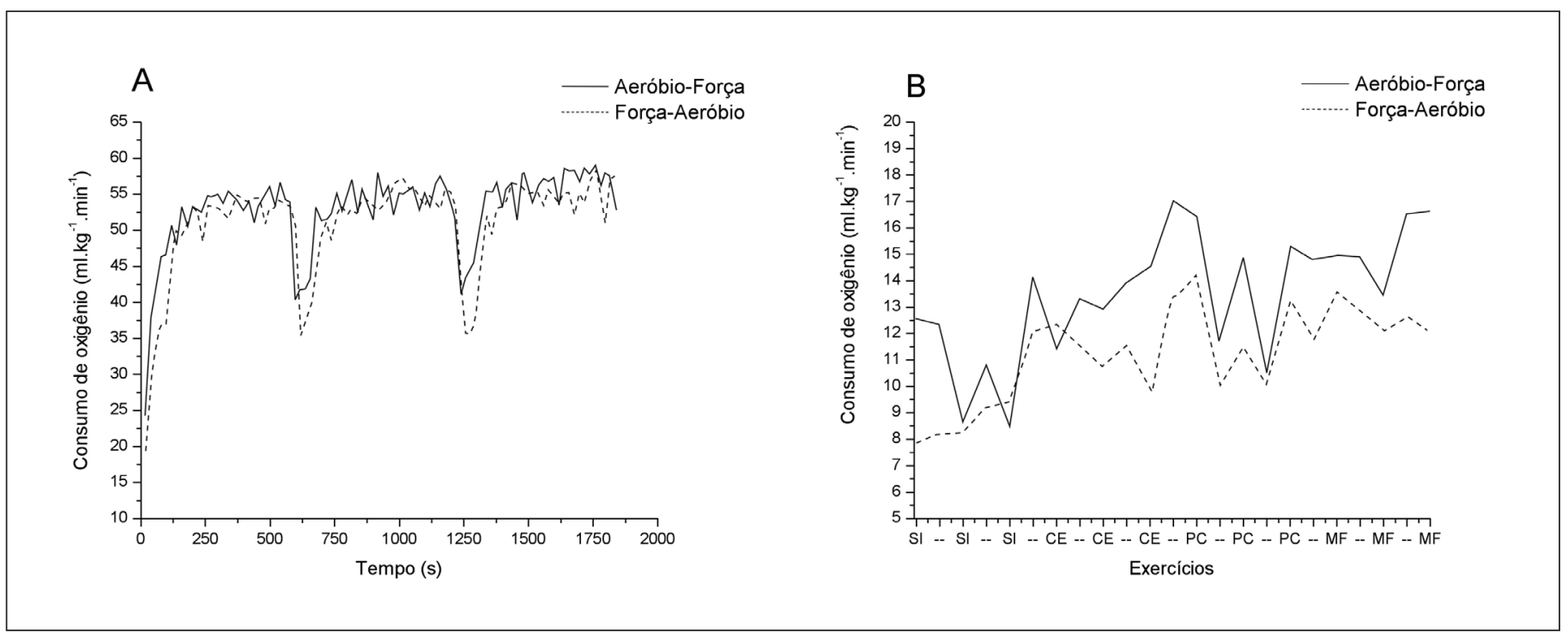

Figura 1. Exemplo típico do comportamento do consumo de oxigênio durante a realização do exercício aeróbio (painel A) e de força (painel B) nas diferentes situações. Onde $\mathrm{SI}$ = supino inclinado; $\mathrm{CE}$ = cadeira extensora; $\mathrm{PC}=$ puxador costas; $\mathrm{MF}=$ mesa flexora.
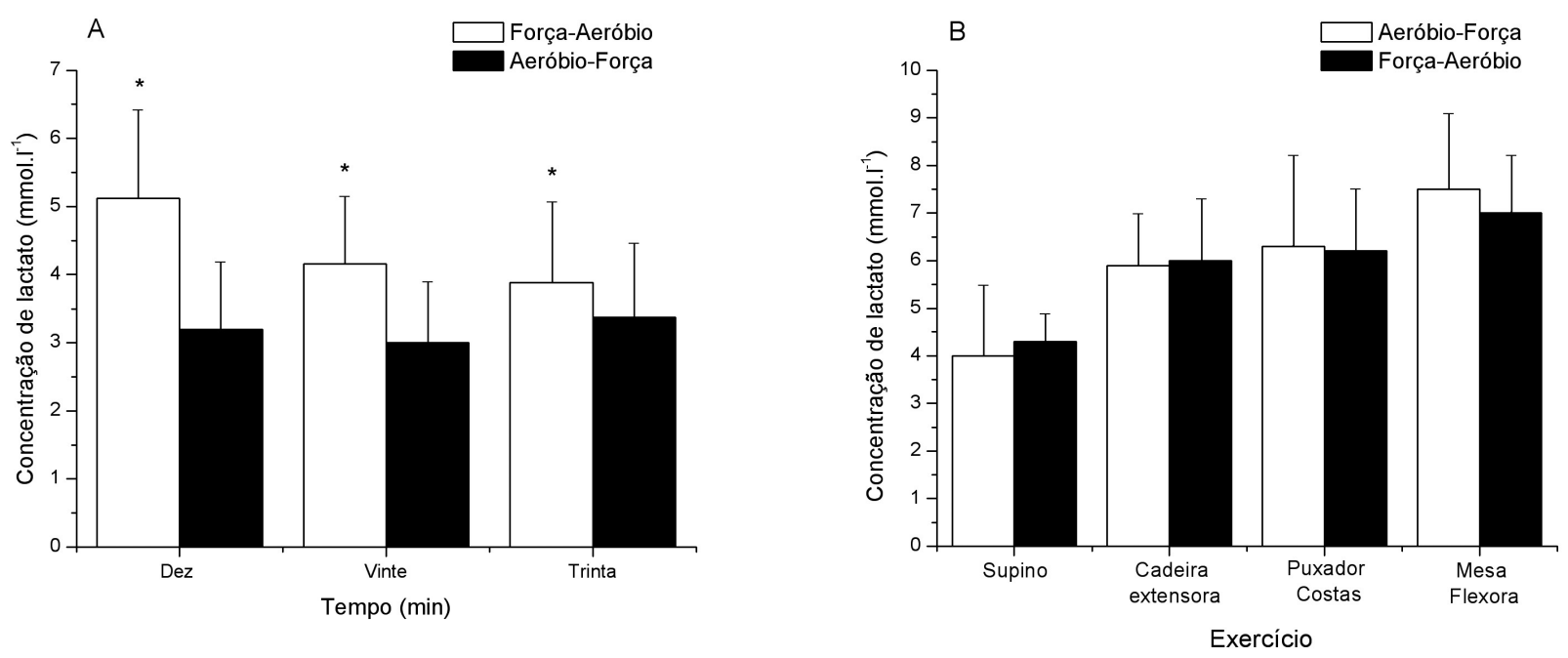

Figura 2. Concentrações sanguíneas de lactato durante treinamento aeróbio (painel A) e de força (painel B) com as diferentes ordens de execução ( $n=10$ ) * = significativamente diferente da ordem aeróbio-força $(p<0,05)$. 


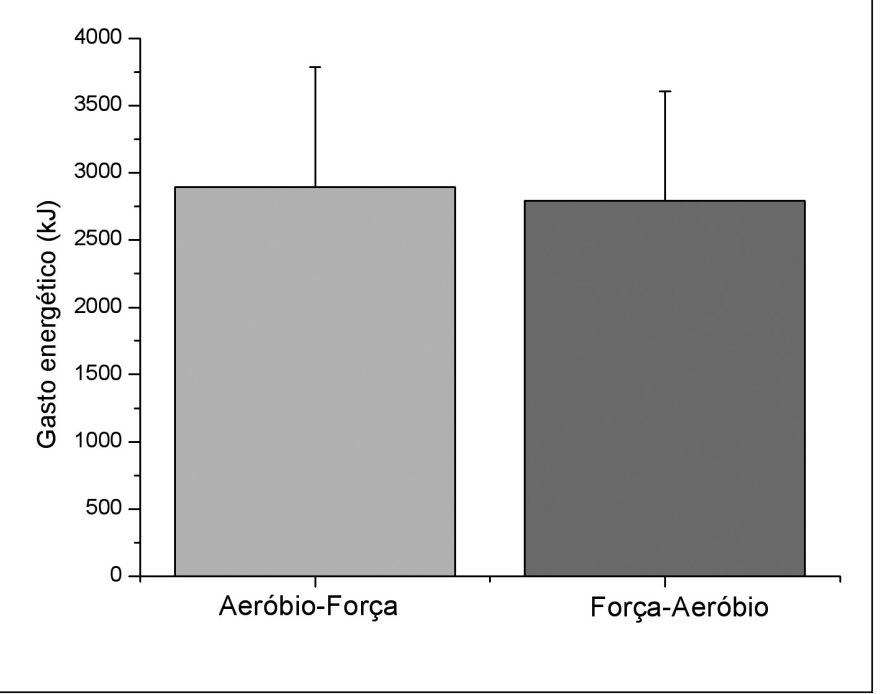

Figura 3. Gasto energético total nas diferentes ordens de execução $(n=10)$

Na tabela 2 estão presentes as repetições realizadas durante os exercícios de força. Não foram constatadas diferenças quanto ao número de repetições realizadas ( $p>0,05$ ), resultando em ausência de diferença $(p>0,05)$ na carga total levantada entre a situação AF $(52,0$ $\pm 7,6$ toneladas $)$ e FA $(53,4 \pm 7,6$ toneladas $)$.

Tabela 2. Média e desvio padrão do número de repetições durante os exercícios de força $(n=10)$.

\begin{tabular}{l|c|c|c|c|c|c}
\hline & \multicolumn{3}{|c|}{ AF } & \multicolumn{3}{c}{ FA } \\
\hline & $\mathbf{1}$ & $\mathbf{2}$ & $\mathbf{3}$ & $\mathbf{1}$ & $\mathbf{2}$ & $\mathbf{3}$ \\
\hline Supino Inclinado & $12 \pm 0$ & $11 \pm 2$ & $9 \pm 3$ & $12 \pm 0$ & $11 \pm 2$ & $10 \pm 3$ \\
\hline Cadeira extensora & $12 \pm 1$ & $12 \pm 1$ & $11 \pm 2$ & $12 \pm 0$ & $12 \pm 1$ & $11 \pm 1$ \\
\hline Puxador costas & $12 \pm 1$ & $12 \pm 2$ & $10 \pm 2$ & $12 \pm 1$ & $11 \pm 1$ & $11 \pm 2$ \\
\hline Mesa flexora & $12 \pm 2$ & $11 \pm 2$ & $10 \pm 3$ & $12 \pm 1$ & $12 \pm 1$ & $11 \pm 2$ \\
\hline
\end{tabular}

\section{DISCUSSÃO}

O principal achado deste estudo foi que não houve diferenças no GE total em decorrência da ordem de execução do exercício concorrente. Contudo, a [La-] permaneceu mais elevada durante a realização do exercício aeróbio nos minutos 10, 20 e 30 na ordem FA, quando comparada com a ordem inversa. Portanto, embora a realização dos exercícios nas diferentes ordens de execução tenha sido capaz de alterar as respostas fisiológicas da atividade subsequente, essas alterações não foram suficientes para ocasionar diferenças no GE total.

Contrapor os resultados deste estudo com os da literatura existente é difícil, uma vez que existem diversos modelos experimentais utilizando TC a curto e a longo prazo, diferindo em relação à frequência, à duração, à intensidade e à aptidão física dos sujeitos ${ }^{(7)}$. Além disso, poucos estudos analisaram os efeitos da ordem de execução sobre o consumo de oxigênio ${ }^{(9,10,20)}$. Por exemplo, Palmer e Sleivert ${ }^{(21)}$ verificaram que $\dot{V}_{2}$ era mais elevado no exercício aeróbio realizado uma e oito horas após o exercício de força em relação à mesma situação sem esforço prévio. Drummond et al. ${ }^{(10)}$ também evidenciaram aumento do $\dot{\mathrm{VO}}_{2}$, da FC e da percepção subjetiva do esforço durante a realização do exercício aeróbio em três períodos de tempo (10, 20 e 25 minutos) da ordem FA quando comparado com a ordem AF, contrapondo aos resultados encontrados por nosso estudo. No entanto, é importante ressaltar que as sessões de treinamento de força utilizadas nesses estudos foram compostas de seis ${ }^{(21)}$ e sete exercícios ${ }^{(10)}$. Portanto, é muito provável que o trabalho total tenha sido maior do que o de nosso estudo. Essa maior quantidade de trabalho total poderia explicar o aumento do $\dot{\mathrm{V}} \mathrm{O}_{2}$ durante a realização da atividade aeróbia na ordem FA no estudo de Drummond et al. ${ }^{(10)}$ e no exercício aeróbio submáximo uma e oito horas após o exercício de força no estudo de Palmer e Sleivert ${ }^{(21)}$.

Por sua vez, Crawford et al. ${ }^{(20)}$ constataram aumento da FC durante os cinco primeiros minutos da realização do exercício aeróbio em relação à situação sem execução prévia de um exercício de força (extensão dos joelhos). Contudo, esse aumento na FC não foi acompanhado por elevação do $\dot{V}_{2}$. Já, Bailey et al. ${ }^{(9)}$ utilizaram um protocolo composto por nove exercícios de força e verificaram que a FC permaneceu elevada por 20 minutos no exercício aeróbio quando comparada com situação controle.

Como não houve interferência da atividade aeróbia no desempenho dos exercícios de força, a ausência de diferença no GE nas diferentes ordens pode ser alternativamente explicada pela similaridade do volume total de treinamento. Segundo Docherty e Sporer ${ }^{(22)}$, a interferência de uma atividade sobre a outra ocorre quando ambas são limitadas pelo mesmo fator (periférico ou central). Recentemente, Souza et al. ${ }^{(23)}$ analisaram o impacto de dois protocolos de exercício aeróbio (acima e abaixo do limiar anaeróbio) sobre o desempenho em uma sessão de treinamento de força a 80\% 1RM. Foi verificado que apenas o exercício aeróbio intenso influenciou negativamente o desempenho na sessão de força, sugerindo que, de fato, a interferência foi decorrente da fadiga de ordem periférica, a qual foi ocasionada por essa atividade intensa prévia. Adicionalmente, no estudo de Souza et al. ${ }^{(23)}$ os participantes executavam o exercício de força a $80 \%$ de 1 RM até a exaustão, ao passo que, em nosso estudo, o número de execuções foi fixado em 12 repetições para uma carga inferior (70\% de 1RM).

No presente estudo, apesar do GE total não apresentar diferenças significativas entre as ordens de execução, houve o aumento do $\dot{\mathrm{V}} \mathrm{O}_{2}$ durante a realização e a recuperação das séries do supino inclinado na ordem AF, quando comparado com a ordem inversa. Isso parece ser decorrente do EPOC, proveniente da atividade anterior, uma vez que o consumo de oxigênio não retorna aos seus níveis de repouso imediatamente após o término da atividade ${ }^{(24)}$. Essa diferença ocorreu somente na ordem AF porque o efeito do EPOC, resultante do exercício de força, foi insuficiente para ocasionar mudanças no $\dot{\mathrm{VO}}_{2}$ da atividade aeróbia, pois durante os exercícios aeróbios em intensidade similar à utilizada em nosso estudo o $\dot{V}_{2}$ é muito maior que durante a realização dos exercícios de força ${ }^{(21,25)}$, fazendo com que o EPOC na ordem FA não ocasione aumento no consumo de oxigênio na atividade aeróbia.

Quanto à concentração de lactato, foi observado que ela permaneceu elevada durante todo o período de exercício aeróbio na ordem FA quando comparado com a ordem AF. Está bem descrito na literatura que o treinamento de força gera altas concentrações de lactato no sangue devido à ativação da via glicolítica ${ }^{(26)}$. Em decorrência desse acúmulo, as [La`] estavam maiores durante o exercício aeróbio na ordem FA, quando comparadas com a ordem AF. Como o exercício aeróbio foi realizado em intensidade inferior à do limiar anaeróbio, é natural se esperar a diminuição das [La'] durante o exer- 
cício aeróbio em virtude da oxidação desse metabólito pelos grupos musculares ativos na corrida ${ }^{(27-30)}$.

\section{CONCLUSÃO}

A realização dos exercícios nas diferentes ordens de execução afetou apenas o consumo de oxigênio no início da sessão de exercício de força (AF) e a [La-] no exercício aeróbio (FA). Contudo, essas alterações fisiológicas não tiveram magnitude suficiente para ocasionar diferenças no gasto energético. Do ponto de vista prático, os resultados do presente estudo permitem indicar que a ordem de execução dos exercícios não é um fator importante para maximizar o gasto energético quando utilizado esse tipo de treinamento nas intensidades estudadas.

\section{AGRADECIMENTOS}

Agradecemos ao Programa Institucional de Bolsas de Iniciação Científica PIBIC/Mackenzie pelo apoio financeiro.

Todos os autores declararam não haver qualquer potencial conflito de interesses referente a este artigo.

\section{REFERÊNCIAS BIBLIOGRÁFICAS}

1. World Health Organization. Obesity: prevening and managing the global epdemic. Geneva: World Health Organization, 1998.

2. Brum PC, Forjaz CL, Tinucci T, Negrão CE. Adaptações agudas e crônicas do exercício físico no sistema cardiovascular. Rev Paul Educ Fís. 2004;18:21-31.

3. Horton TJ, Hill J. Exercise and obesity. Proceed Society Nutr. 1990;57:85-91.

4. Pollock ML, Gaesser GA, Butcher JD, Després JP, Dishman RK, Franklin B A, et al. The recommended quantity and quality of exercise for developing and maintaining cardiorespiratory and muscular fitness, and flexibility in healthy adults. Med Sci Sports Exerc. 1998;30(6):975-91.

5. Holloszy JO, Coyle EF. Adaptations of skeletal muscle to endurance exercise and their metabolic consequences. J Appl Physiol. 1984;56(4):831-8. Review.

6. MacDougall JD, Elder GC, Sale DG, Moroz JR, Sutton JR. Effects of strength training and immobilization on human muscle fibres. Eur J Appl Physiol Occup Physiol. 1980;43(1):25-34.

7. Leveritt M, Abernethy PJ, Barry BK, Logan PA. Concurrent strength and endurance training: a review. Sports Med. 1999;28(6):413-27. Review.

8. Hickson RC. Interference of strength development by simultaneously training for strength and endurance. Eur J Appl Physiol Occup Physiol. 1980;45(2-3):255-63.

9. Bailey ML, Khodiguian N, Farrar P. Effects of resistance exercise on selected physiological parameters during subsequent aerobic exercise. J Strength Cond Res. 1996;10(2):101-4.

10. Drummond MJ, Vehrs PR, Schaalje GB, Parcell AC. Aerobic and resistance exercise sequence affects excess postexercise oxygen consumption. J Strength Cond Res. 2005;19(2):332-7.

11. Scott CB. Contribution of blood lactate to the energy expenditure of training. J Strength Cond Res. 2006;20(2):404-11

12. Beneke R, Beyer T, Jachner C, Erasmus J, Hutler M. Energetics of karate kumite. Eur J Appl Physiol. 2004;92(4-5):518-23.

13. Heck H, Mader A, Hess G, Mucke S, Muller R, Hollmann W. Justification of the 4-mmol// lactate threshold. Int J Sports Med. 1985;6(3):117-30.

14. Astorino TA, Rietschel JC, Tam PA, Taylor K, Johnson SM, Freedman TP, et al. Reinvestigation of optimal duration of $\mathrm{VO}_{2}$ max testing. J Exerc Physiology. 2004;7(6):1-8.

15. Brown LE, Weir JP. Recomendações de procedimentos da Sociedade Americana de fisiologia do exercício (ASEP) I: avaliação precisa da força e potência muscular. Rev Bra Cien Mov. 2003;11(4):95-110.
16. Melby C, Scholl C, Edwards G, Bullough R. Effect of acute resistance exercise on postexercise energy expenditure and resting metabolic rate. J Appl Physiol. 1993;75(4):1847-53.

17. Beneke R, Pollmann C, Bleif I, Leithauser RM, Hutler M. How anaerobic is the Wingate anaerobic test for humans? Eur J Appl Physiol. 2002;87(4-5):388-92.

18. Margaria R, Edwards HT, Dill DB. The possible mechanism of contracting and paying the oxygen debt and the role of lactic acid in muscular contraction. Am J Physiol. 1933;106:689-714.

19. Di Prampero PE, Ferretti G. The energetics of anaerobic muscle metabolism: a reappraisal of older and recent concepts. Respir Physiol. 1999;118(2-3):103-15. Review.

20. Crawford WW, Loy SF, Nelson AG, Conlee RK, Fisher AG, Allsen PE. Effects of prior strength exercise on the heart rate oxygen uptake relationship during submaximal exercise. J Sports Med Phys Fitness. 1991;31(4):505-9.

21. Palmer CD, Sleivert GG. Running economy is impaired following a single bout of resistance exercise. J Sci Med Sport. 2001;4(4):447-59.

22. Docherty D, Sporer B. A proposed model for examining the interference phenomenon between concurrent aerobic and strength training. Sports Med. 2000;30(6):385-94.

23. Souza EO, Tricoli V, Franchini E, Paulo AC, Regazzini M, Ugrinowitsch C. Acute effect of two aerobic exercise modes on maximum strength and strength endurance. J Strength Cond Res. 2007;21(4):1286-90

24. Borsheim, E, Bahr R. Effect of exercise intensity, duration and mode on post-exercise oxygen consumption. Sports Med. 2003;33(14):1037-60.

25. Tomlin DL, Wenger HA. The relationship between aerobic fitness and recovery from high intensity intermittent exercise. Sports Med. 2001;31(1):1-11.

26. MacDougall JD, Ray S, Sale DG, McCartney N, Lee P, Garner S. Muscle substrate utilization and lactate production during weightlifting. Can J Appl Sport Sci. 1999;24(3):209-15

27. Baldari C, Videira M, Madeira F, Sergio J, Guidetti L. Blood lactate removal during recovery at various intensities below the individual anaerobic threshold in triathletes. J Sports Med Phys Fitness. 2005:45(4):460-6.

28. Gladden LB. Muscle as a consumer of lactate. Med Sci Sports Exerc. 2000;32(4):764-771

29. Brooks GA. The lactate shuttle during exercise and recovery. Med Sci Sports Exerc. 1986;18(3):360-368. 30. Oosthuyse T, Carter RN (1999). Plasma lactate decline during passive recovery from high-intensity exercise. Med Sci Sports Exerc. 1999;31(5):670-674 